\title{
MMIC front-ends for optical communication systems
}

\section{Petersen, Anders Kongstad}

\section{Published in:}

Proceedings of the Sixth Annual IEEE International ASIC Conference and Exhibit

Link to article, DOI:

10.1109/ASIC.1993.410765

Publication date:

1993

Document Version

Publisher's PDF, also known as Version of record

Link back to DTU Orbit

\section{Citation (APA):}

Petersen, A. K. (1993). MMIC front-ends for optical communication systems. In Proceedings of the Sixth Annual IEEE International ASIC Conference and Exhibit (pp. 486-489). IEEE. https://doi.org/10.1109/ASIC.1993.410765

\section{General rights}

Copyright and moral rights for the publications made accessible in the public portal are retained by the authors and/or other copyright owners and it is a condition of accessing publications that users recognise and abide by the legal requirements associated with these rights.

- Users may download and print one copy of any publication from the public portal for the purpose of private study or research.

- You may not further distribute the material or use it for any profit-making activity or commercial gain

- You may freely distribute the URL identifying the publication in the public portal

If you believe that this document breaches copyright please contact us providing details, and we will remove access to the work immediately and investigate your claim 


\title{
MMIC FRONT-ENDS FOR OPTICAL COMMUNICATION SYSTEMS
}

\author{
Anders K. Petersen \\ Technical University of Denmark, \\ Center for Broadband Telecommunications \\ Bldg. 348, DK-2800 Lyngby, Denmark
}

\begin{abstract}
This paper presents two different types of optical front-end MMIC amplifiers for a $2.5 \mathrm{Gbit} / \mathrm{s}$ coherent heterodyne optical receiver. A bandwidth of 6-12 GHz has been obtained for a tuned front-end and 3-13 $\mathrm{GHz}$ for a distributed front-end. An input noise current density of 5-15 pA $/ \sqrt{\mathrm{Hz}}$ has been obtained for the tuned front-end, which is the lowest value reported in this frequency range for a GaAs MESFET front-end.
\end{abstract}

\section{Introduction}

Optical communication systems based on coherent heterodyne detection have been studied intensively during the last few years. In a coherent optical communication system the light from a laser (normally a semiconductor laser) is used as a carrier. There are many ways to modulate the light, one of the most efficient ways at several Gbit/s is by using continuous phase frequency shift keying (CP-FSK). CPFSK signals can be generated efficiently by directly modulating the current of a semiconductor laser with a digital baseband signal. The frequency of a semiconductor laser changes slightly when the injection current of the laser is changed. Modulating the current with a binary signal, one frequency represents a binary " 0 " and a second frequency represents a binary " 1 ". System experiments based on this scheme has been published in a numerous amount of papers [1], [2], [3].

In a coherent optical receiver the same principles are used as in radio communications. In the following the operation of the receiver is briefly described. Fig. 1 shows a diagram of a receiver for a CP-FSK system. At the left hand an optical signal is received through an optical fibre from a distant point, the optical signal is combined in a fibre coupler with a laser operating as a local oscillator. The combined optical signals are detected by the photodiode. During this detection the signal is downconverted to an intermediate frequency corresponding to the frequency difference between the local oscillator and the frequency of the lightwave carrier. The wavelength of the lasers are typically around $1.5 \mu \mathrm{m}$, corresponding to approximately $200 \mathrm{THz}$. The front-end has two main functions in the receiver, first it acts as a low noise amplifier, second it converts the photodiode current into a voltage at the output. In the receiver in fig. 1 the signal is further amplified by IF amplifiers. After IF amplification the signal is demodulated, and the original baseband signal is regenerated. After demodulation the baseband signal can be treated as in any conventional communication system.

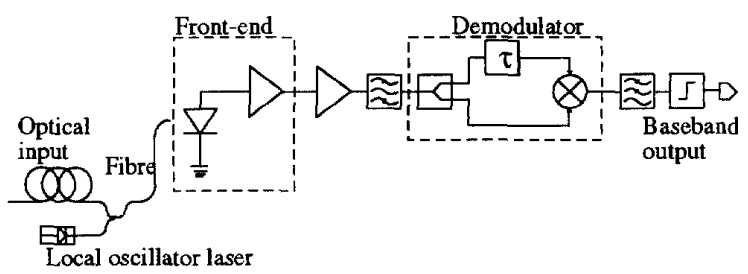

Fig. 1. Block diagram of a coherent optical CP-FSK receiver

One of the critical components in the coherent optical receiver is the optical front-end. The front-ends presented in this paper are designed for a $2.5 \mathrm{Gbit} / \mathrm{s}$ CP-FSK system operating at an IF of $9 \mathrm{GHz}$. An important parameter in a CP-FSK receiver is the modulation index, $\beta$, which is defined as

$$
\beta=\frac{\left|f_{1}-f_{0}\right|}{B}
$$

where $f_{1}$ and $f_{0}$ are the two frequencies at IF level representing binary " 1 " and " 0 " and $B$ is the bit rate. The optimum value of $\beta$ is in the range $0.6-0.8$ [4]. With such values bandwidths of approximately two times the bit rate are required, which at $2.5 \mathrm{Gbit} / \mathrm{s}$ requires a minimum bandwidth of $5 \mathrm{GHz}$ around an IF of $9 \mathrm{GHz}$. Several different front-end topologies have been published, mainly hybrid integrated circuits based on advanced technologies such as HEMTs [5], [6], [7]. In the following sections two 
different MMIC front-end topologies, which can be applied in a coherent heterodyne receiver, are presented and compared.

\section{Distributed front-end}

The distributed amplifier has been suggested as an optical low-noise front-end amplifier in [8], [9]. By using this technique the gain-bandwidth can be increased compared to resonant amplifiers. A simplified schematic diagram of the distributed amplifier is shown in fig. 2. The amplifier consists of two artificial transmission lines. The gate line includes the inductive elements $\lg _{g} \log _{g}$, the gate source capacitances of the four FETs and the junction capacitance of the photodiode. The parasitics from the FETs and the photodiode are in this way incorporated in the artificial gate transmission line. The gate line is terminated in $\mathrm{Z}_{\mathrm{g}}$ to avoid reflections. The second transmission line incorporates the drain source capacitances in the drain line that also includes $\mathbf{l}_{\mathrm{d} 1} \mathbf{l}_{\mathrm{d} 5}$, this line is also terminated to avoid reflections.

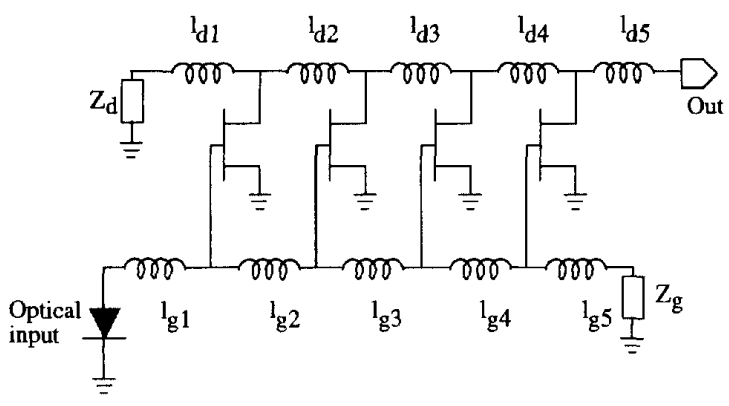

Fig. 2. Schematic circuit diagram of distributed front-end amplifier

The distributed amplifier has been realised as an MMIC (Monolithic Microwave IC) using the ion implanted GaAs MESFET foundry proces of GEC Marconi with an $\mathbf{f}_{\mathrm{T}}$ of 20 $\mathrm{GHz}$. This MMIC includes the circuit shown in fig. 2 except for the photodiode and $l_{\mathrm{g} 1}$, which is realised as the bond wire between the photodiode and the MMIC.

The layout of the MMIC is shown in fig. 3. The sources of the four FETs in the amplifier are grounded using on-chip via holes. The two artificial transmission lines appear clearly from the figure, the inductors forming $l_{\mathrm{g} 2}-\mathrm{l}_{\mathrm{g}}$ and

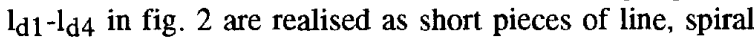
inductors or a combination of both.

The S-parameters of the chips have been measured using on-wafer probes. Fig. 4 shows measured and simulated $\left|S_{21}\right|$ for three MMICs in the frequency range $0-20 \mathrm{GHz}$. The figure shows a close agreement between the theoretical and experimental results. The deviation between the different samples also appears in the figure and is typical below \pm 1 $\mathrm{dB}$ over the entire pass band.

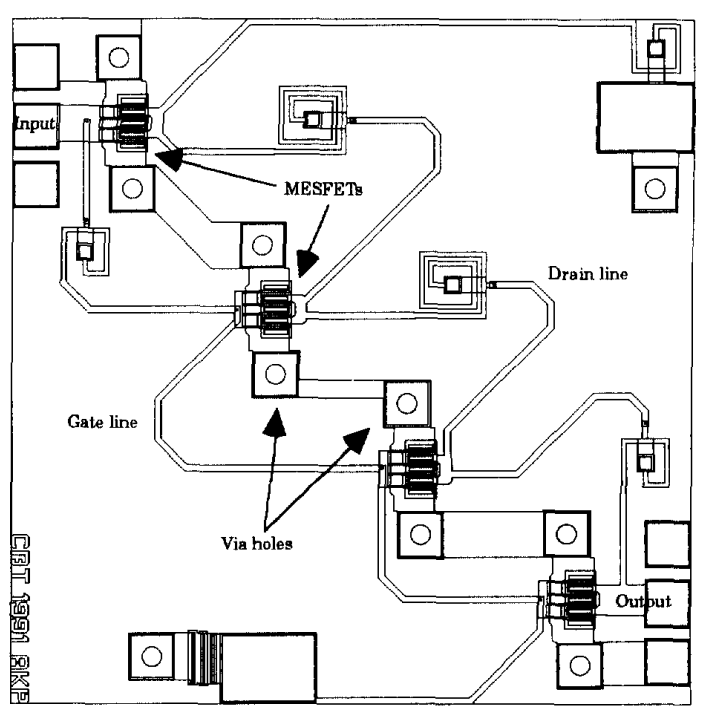

Fig. 3. Layout of distributed MMIC amplifier. The chip size is $2 \times 2 \mathrm{~mm}$.

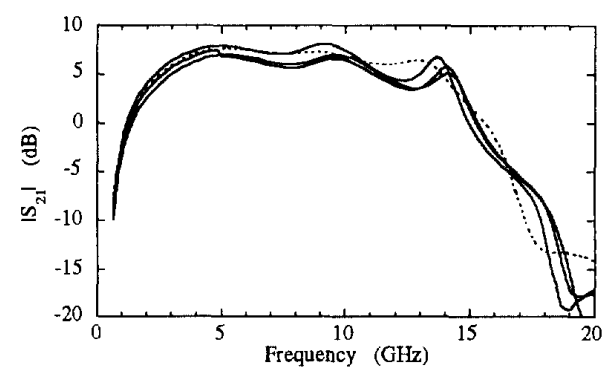

Fig. 4. Simulated and on-wafer measured transmission $\left(\left|S_{21}\right|\right)$ of distributed amplifier MMIC. Dashed curve is simulated and solid curves are measurements of three different samples.

The MMIC was mounted together with a wide band GaInAs/InP p-i-n photodiode on alumina thinfilm substrate. A photodiode based on InP was chosen because the wavelength in optical telecommunication systems is 1.3-1.6 $\mu \mathrm{m}$. The transimpedance and the equivalent input noise current density were measured for the complete front-end. The noise performance was measured using the method described in [10] in the frequency range from 5-18 GHz and the transimpedance was measured using a lightwave test-set (HP83420A). The results of these measurements are shown in fig. 5 .

Fig. 5 shows excellent agreement between the measurements and simulation except for a dip in the transimpedance around $9 \mathrm{GHz}$. The target transimpedance of $38 \mathrm{~dB} \Omega \pm 1 \mathrm{~dB} \Omega$ was obtained over a $3-13 \mathrm{GHz}$ 


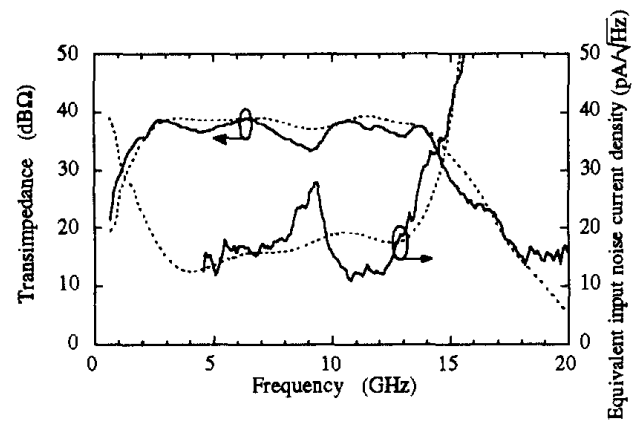

Fig. 5. Measured and simulated transimpedance and equivalent input noise current density of distributed front-end. The dashed curves are simulated and the solid curves are measurements.

bandwidth except for the dip. This dip is assumed to be caused by the bias circuitry of the FET gates and leads to an increase in the input noise current density.

\section{Transformer tuned front-end}

The transformer tuned front-end was first suggested in [11]. A simplified schematic circuit diagram of the transformer tuned front-end presented in this paper is shown in fig. 6. The technique in the tuned front-end is to form a resonant circuit between the parasitic capacitances, e.g. the photodiode junction capacitance and the gate source capacitance of the FET in the first stage. When transformer tuning is applied a T-equivalent circuit can be used. In fig. 6 such a T-equivalent circuit is shown consisting of $\mathrm{l}_{\mathrm{b} 1}-\mathrm{l}_{\mathrm{b} 3}$. By appropriate choice of the inductors in the tuning circuits a band-pass amplifier is obtained, which is desirable in a heterodyne receiver.

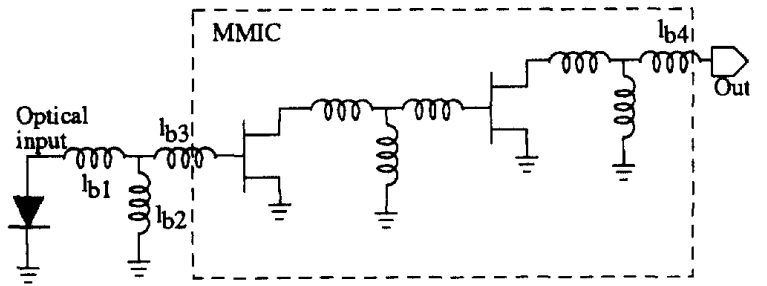

Fig. 6. Simplified schematic circuit diagram of tuned front-end.

Extreme care should be taken when a tuned amplifier is designed, because of the high $Q$ resonant circuits. This is especially the case when integrated as a monolithic circuit, since adjustments are impossible or very limited. One of the advantages on the other hand is that the inductive elements used in the tuning are much easier to control on-chip.

The components within the dashed box in fig. 6 have been integrated in an MMIC. The layout of the MMIC is shown in fig. 7.

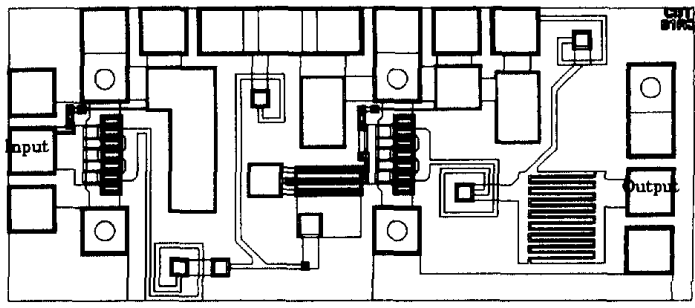

Fig. 7. Layout of tuned MMIC amplifier. The chip size is $1 \times 2 \mathrm{~mm}$.

In this design the values of the inductors in the tuning circuits are very critical, it is therefore important that a welldefined ground is available on-chip for the tuning circuits, in particular the shunt inductor in the T-equivalent circuit. An interdigital capacitor has also been used in the output tuning network, since the tolerances on this type of capacitor is very small.

The MMICs for the tuned front-end have also been characterised on-wafer and compared with simulations. Fig. 8 shows the transmission in a $50 \Omega$ system. It is observed from this figure that excellent agreement was obtained, indicating that it is indeed possible to design well-defined tuning elements. This figure also shows that the deviation between the individual samples measured are very small.

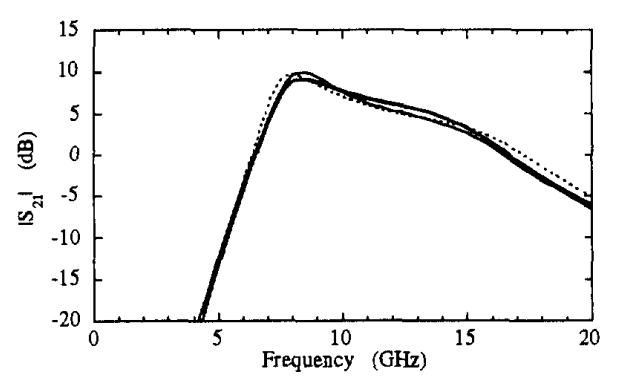

Fig. 8. Simulated and on-wafer measured transmission $\left(\left|\mathbf{S}_{21}\right|\right)$ of transformer tuned MMIC amplifier. Dashed curve is simulation and solid curves are measurements.

The transmission is not flat over frequency, since it has been characterised in a $50 \Omega$ system. When the MMIC is used together with a photodiode as shown in fig. 6 a relatively flat response is obtained.

The tuned MMIC amplifier has also been mounted with a photodiode on alumina substrate. The tuning circuit between the photodiode and the MMIC was realised as bond wires. This tuning circuit was left outside the MMIC to have a possibility of making minor adjustments after the MMIC was manufactured. 
The transimpedance and equivalent input noise current density were measured in the same way as the distributed front-end. The results of these measurements are shown in fig. 9 .

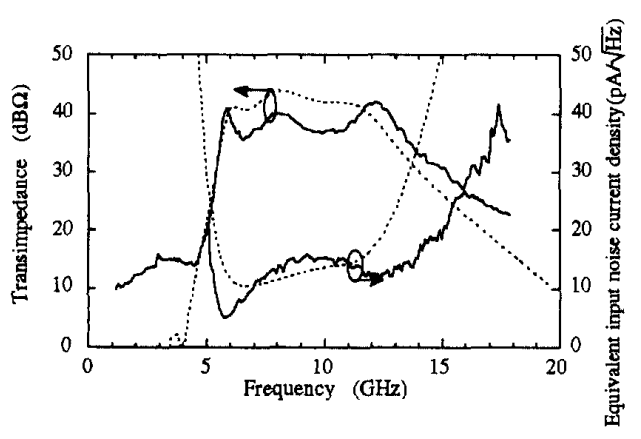

Fig. 9. Measured and simulated transimpedance and equivalent input noise current density of tuned front-end. Dashed curves are simulations and solid curves are measurements.

A very low input noise current density of $5-15 \mathrm{pA} / \sqrt{\mathrm{Hz}}$ was obtained over the frequency range $5-14 \mathrm{GHz}$. Both the noise and transimpedance are in good agreement with the simulations. However, the ripple is somewhat larger than expected, the transimpedance falls between $36-42 \mathrm{~dB} \Omega$ in the frequency range 6-12 GHz. Since the agreement between simulations and measurements of the MMIC itself was very good, the explanation has to be found in the bond wire tuning network between the photodiode and the MMIC,

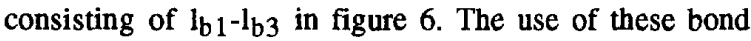
wires introduces some unpredictable effects because the length and shape of the bond wires are difficult to model.

\section{Discussion}

The previous sections clearly demonstrate that the bandwidth of the distributed front-end is much higher than the tuned front-end, the transimpedance of the two amplifier types is approximately the same. The price to pay for larger bandwidth is the input noise current density, which is slightly higher for the distributed front-end compared to the tuned front-end. The transimpedance level for both front-ends is around $39 \mathrm{~dB} \Omega$, this is not a state-of-the-art result, but could be improved considerably by using more advanced processes such as HEMT processes.

The agreement between measured and simulated Sparameters for the MMICs indicates that well-defined circuit elements are possible to realise on-chip. On the other hand the introduction of bond wires makes it more difficult to obtain good agreement, because they are difficult to control and model.

Compared to hybrid integrated circuits that are using bond wires in large scale, the MMIC solution offers better performance and lower price because of mounting of fewer components.

\section{Conclusions}

Two different optical front-end amplifiers for a $2.5 \mathrm{Gbit} / \mathrm{s}$ optical heterodyne CP-FSK receiver have been presented. The two front-ends are a transformer tuned front-end and a distributed front-end. The front-ends were realised in a lowcost commercially available ion-implanted GaAs MESFET process with an $\mathrm{f}_{\mathrm{T}}$ of $20 \mathrm{GHz}$. A bandwidth of $6-12 \mathrm{GHz}$ and $3-13 \mathrm{GHz}$ was demonstrated for the tuned and distributed front-end, respectively. The noise current density for the tuned front-end was 5-15 pA/ $\sqrt{\mathrm{Hz}}$ and $10-28 \mathrm{pA} / \sqrt{\mathrm{Hz}}$ for the distributed front-end. Very good agreement was demonstrated between the simulated and measured Sparameters of both MMICs. The largest discrepancies were observed when the MMICs were mounted together with the photodiode. These discrepancies were due to the poor control over external parasitics, such as bond wires.

\section{Acknowledgements}

The author would like to thank Dr. F. Ebskamp for many fruitful discussions during the design and test of the frontends and in particular A. M. Jagd for designing the transformer tuned MMIC. The author would also like to thank Inger Hansen, Elektronik Centralen, for bonding of the chips.

\section{References}

[1] T. Sugie, T. Imai and T. Ito, "Over $350 \mathrm{~km}$ CPFSK repeaterless transmission at $2.5 \mathrm{Gbit} / \mathrm{s}$ employing high-output power erbiumdoped fibre amplifiers", Electron. Lett., Vol. 26, pp. 1577-1578, 1990.

[2] T. Chikama et al. "Optical heterodyne continuous phase FSK transmission experiment up to $4 \mathrm{Gbit/s",} \mathrm{Proc.} \mathrm{of} \mathrm{ECOC} 90$, Vol. 1, pp. 65-68, Amsterdam 1990.

[3] S. Yamazaki, "Compensation for modulational instability in highly dispersive coherent optical repeater transmission systems", Proc. of CLEO 92, Paper CMB4, pp. 10-12, Anaheim 1992.

[4] G. Jacobsen and K. Emura, "Performance of CP-FSK systems with tight IF filtering and optimised delay time", IEE Proc.-J, Vol. 139 , pp. 158-162, 1992.

[5] N. Ohkawa, "20 GHz low-noise HEMT preamplifier for optical receivers", Proc. of ECOC, Vol. 1, pp. 404-407, 1988.

[6] J. L. Gimlett, "A new low noise 16 GHz PIN/HEMT optical receiver", Proc. of ECOC, Vol. 2, pp. 13-16, 1988.

[7] F. Ebskamp, R. J. S. Pedersen and G. Schiellerup, "A balanced 6$15 \mathrm{GHz}$ low-noise tuned optical front-end for multi-gigabit-persecond heterodyne system applications", Proc. of CLEO, paper CTuL5, pp. 172-174, 1992.

[8] C. S. Aitchison, "Potential of the distributed amplifier as a photodiode detector amplifier in high bit rate optical communication systems", Electron. Lett., vol. 26, pp. 1693-1695, 1990.

[9] N. Takachio, "A 10 Gbit/s optical heterodyne detection experiment using a $23 \mathrm{GHz}$ bandwidth balanced receiver", Proc. IEEE MTT Symposium, paper C-2, pp. 149-151, 1990.

[10] A. K. Petersen and A. Jagd, "Tuned optical front-end MMIC amplifiers for a coherent optical receiver", Proc. of European Microwave Conference, pp. 1065-1070, 1992.

[11] G. Jacobsen, J. X. Kan and I. Garrett, "Tuned front-end design for heterodyne optical receivers", J. Lightwave Technology, vol. 7, pp. 105-114, 1989. 